\title{
EMPRESA PRIVADA: PRINCIPAL SOCIO EN EL POSCONFICTO Y LA CONSTRUCCIÓN DE PAZ
}

\author{
Private Companies: Post-conflict and Peace-building Main Partner
}

Empresa privada: principal sócio no pós-conflito e a construção de paz

RECIBIDO: 20 DE NOVIEMBRE DE 2015

es

\section{RESUMEN}

El objetivo de este trabajo es desarrollar una reflexión sobre el papel de la empresa privada en el posconflicto, la construcción de paz en Colombia y los mecanismos que tiene para desempeñarlo. La metodología utilizada es documental y toma como eje fundamental la teoría ecléctica de construcción de paz.

Los principales resultados señalan que la empresa privada tiene un papel clave en el proceso de construcción de paz, los empresarios entienden que un país sin guerra mejora las posibilidades de negocios y que para contribuir efectivamente a su desarrollo existen herramientas jurídicas y organizacionales, como la responsabilidad social empresarial, las alianzas estratégicas con el Gobierno y como financiadora de paz. Sin embargo, gran parte del sector privado desconoce estos instrumentos y por tanto su desempeño no puede ser el ideal. Se concluye que la empresa privada es un actor fundamental en la construcción de paz, pero para su cabal desempeño se requiere cohesionar sus esfuerzos con los de otros actores de la sociedad.

PALABRAS CLAVE: construcción de paz, empresa privada, herramientas de construcción de paz.
EVALUADO: 12 DE FEBRERO DE 2016

Julio Alfonso González Mendoza

(Colombia)

Magíster en Planeación

Doctor en Educación (c)

Universidad Francisco de Paula Santander

alfonsogonzalez@ufps.edu.co

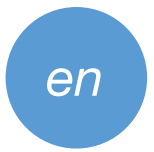

\section{oor}

\section{ABSTRACT}

This paper is a reflection on the role private companies have in a post-conflict scenario and peace-building in Colombia and on the mechanisms there are to carry it out. The methodology used is documentary, based on the reflection of the eclectic theory of peace-building.

The main results indicate that private companies play a key role within the process of peace-building. Entrepreneurs understand that a country without wars improves the possibilities for doing business and that there are legal and organizational tools to effectively contribute to this development, such as Business Social Responsibility, strategic alliances with the government, and as peace financial backer. However, the majority of the private sector are not familiar with these instruments and for this reason, their performance cannot be the ideal one. To conclude, private companies are essential actors of peace-building, but it is necessary to unite forces with other actors of society for it to work out

KEYWORDS: Peace-building, private companies, peace-building tools.
ACEPTADO:30 DE MARZO DE 2016

\section{RESUMO}

O objetivo deste trabalho é desenvolver uma reflexão acerca do papel da empresa privada no pós-conflito e a construção de paz na Colômbia e sobre os mecanismos que tem para desempenhá-lo. A metodologia utilizada é documental e toma como eixo fundamental de reflexão a teoria de eclética de construção de paz.

Os principais resultados assinalam que a empresa privada tem um papel chave no processo de construção de paz, os empresários entendem que um país sem guerra melhora as possibilidades de negócios e que para contribuir efetivamente ao seu desenvolvimento existem ferramentas jurídicas e organizacionais como a Responsabilidade Social Empresarial, Alianças estratégicas com o governo e como financiadora de paz. Porém grande parte de sector privado desconhece estes instrumentos e por tanto o seu desempenho não pode ser o ideal. Conclui-se que a empresa privada é um ator fundamental na construção de paz mas para o seu cabal desempenho requer-se unir os seus esforços com os de outros atores da sociedade.

PALAVRAS CHAVE: construção de paz, empresa privada, ferramentas de construção de paz.

PARA CITAR ESTE ARTÍ́CULO/TO CITE THIS ARTICLE/PARA CITAR ESTE ARTIGO:

González Mendoza, J. A. (2016). Empresa privada: principal socio en el posconflicto y la construcción de paz. Panorama, 10(18) pp. 84-92 . 


\section{INTRODUCCIÓN}

"No hay empresas exitosas en comunidades deterioradas" (Drucker, 2002, p. 94) es una premisa que entienden los empresarios colombianos y por tanto saben que es necesario resolver el conflicto armado para encontrar condiciones favorables a fin de desarrollar negocios prósperos y sostenibles. El sector productivo es consciente que su aporte es valioso en la etapa del posconflicto y la construcción de paz, pero no todos conocen los mecanismos para vincularse efectivamente. El objetivo de este trabajo es reflexionar acerca del rol de la empresa privada en el posconflicto y la construcción de paz en Colombia y de las herramientas disponibles para articular sus esfuerzos con el Estado y los demás actores de la sociedad.

Para el desarrollo del tema, inicialmente se contextualizarán las diferentes perspectivas de construcción de paz, a saber: 1) minimalista, que la define como el proceso de reparar los daños de la guerra, atender a las víctimas y reconstruir la infraestructura, 2) maximalista, que cree que la construcción de paz no solo se trata de arreglar los daños, sino que además es necesario resolver las estructuras sociales que dan origen a las causas del conflicto y 3) ecléctica, en la que la construcción de paz se debe iniciar antes que cesen las hostilidades y culminar cuando la sociedad se ha recuperado del daño físico causado en el transcurso del conflicto, ha aprendido a jugar con nuevas reglas sociales, políticas y económicas y ha sanado sus heridas individuales y colectivas.

A continuación, se explicará el rol de la empresa en el posconflicto y la construcción de paz, en el que se analizan varias posiciones: 1) la empresa como perpetuadora del conflicto, según la cual, debido a su accionar, se concentra riqueza en unos pocos lo cual genera inequidad social, principal causa del conflicto; 2) la empresa como generadora de empleo y oportunidades como quiera que más de $90 \%$ del empleo lo genera el sector privado, y 3) la empresa como víctima, en la que se explica que el mayor perjudicado ha sido el sector, debido a la extorsión y a que ha tenido que vivir en un ambiente inseguro $\mathrm{y}$ con desventajas competitivas frente a sus similares de otros países.

Finalmente, se examinan las herramientas más importantes que tienen los empresarios para involucrarse en el proceso: 1) como financiador de paz, 2) estableciendo alianzas con el Estado y organismos nacionales e internacionales para canalizar recursos, experiencias e ideas y 3) ejerciendo responsabilidad social empresarial.

Las principales conclusiones de esta reflexión señalan que en cualquiera de las perspectivas la empresa tiene un papel fundamental en la construcción de paz, es consciente de su rol, quiere ayudar y está comprometido en ello, pero no todos los empresarios conocen la forma de realizarlo.

\section{EL ROL DE LA EMPRESA PRIVADA}

Al tratar el tema de la paz en Colombia, se encuentra que se ha sido explicado y analizado recurrentemente por muchos teóricos, desde diversas perspectivas, y hasta se llega a creer que realizar un estudio más al respecto no pasa de ser una declaración de intención, ya que la totalidad de la población colombiana ha vivido la mayor parte de su vida, si no toda, en estas condiciones $y$, tal vez, en los imaginarios colectivos solo existe esta forma de convivencia social.

Puede parecer extraño y utópico alcanzar la paz, ya que son cincuenta años de conflicto, el más largo de la era moderna, que deja un saldo trágico no solo en vidas sino en desarrollo, educación, oportunidades y sueños frustrados de toda una generación; sin embargo, es necesario pensar que no hay conflicto que no se pueda resolver y que cualquier estrategia o acción que se emprenda no está demás si puede contribuir a la construcción de paz.

Lograr el fin del conflicto es entrar en una etapa en la que deben ser resueltos los temas que dan origen a la confrontación y en la que el Estado y la sociedad civil deben hacer su mejor esfuerzo para establecer reglas de juego suficientemente efectivas, que logren ser aceptadas por las partes. "El posconflicto es el período de tiempo que sigue a la superación total o parcial de los conflictos armados" (Universidad del Rosario, 2014, p. 1), "es aquel período de tiempo que se inicia con el cese de hostilidades entre las partes previamente enfrentadas" (Rettberg, 2003, p. 20) y en el que se resuelven los temas estructurales de la sociedad de tal forma que garanticen la no repetición del conflicto.

Los esfuerzos del Estado no son suficientes para resolver todos los problemas, por lo que es necesario la
| Empresa privada: principal socio en el posconficto y la construcción de paz
I Panorama I pp. 84-92 I Volumen 10 I Número 18 I Enero-junio I 2016 
Julio Alfonso

González Mendoza I

participación activa de los distintos actores de la sociedad que aporten recursos, ideas y experiencias en una forma coordinada y articulada (Garzón, Parra y Pineda, 2003). La ayuda no solo constituye el suministro de bienes y servicios, sino que, ante todo, consiste de una actuación ética y humanitaria que no discrimine a los destinatarios y reivindique la igualdad entre los seres humanos.

Esta lógica de establecer reglas de juego que la sociedad acepte y que permitan eliminar las diferencias es conocida como "construcción de paz", que la ONU (1992) define como un conjunto de "medidas destinadas a individualizar y fortalecer estructuras que tiendan a reforzar y consolidar la paz a fin de evitar una reanudación del conflicto" (p. 6). En ese periodo de construcción de paz, la sociedad establece un conjunto de políticas, estrategias y acciones para el fortalecimiento de las estructuras sociales, económicas y políticas que logren prevenir y resolver los conflictos sin recurrir al uso de la violencia.

\section{PERSPECTIVA DE CONSTRUCCIÓN DE PAZ}

Cualquiera de las perspectivas del conflicto requiere acciones decididas y coordinadas de los distintos actores sociales para erradicar las casusas de la confrontación y lograr que la sociedad acepte y respete las nuevas reglas de juego e impida que las diferencias de pensamiento e imaginarios sociales sean el origen de nuevos enfrentamientos.

Como eje de reflexión sobre el rol de la empresa privada en el posconflicto y la construcción de paz, en este trabajo se asumirá la perspectiva ecléctica, ya que toma presupuestos válidos de las demás y se extiende por un periodo que va desde antes del fin del conflicto y termina cuando la sociedad acepta las nuevas reglas sociales, lo cual permite una amplia discusión y reflexión,

Panorama I pp. 84-92। Volumen 101 Número 18 । Enero-junio I 2016 I

El esfuerzo para la construcción de paz no es solo responsabilidad de los actores en conflicto, sino que involucra a la sociedad civil entendida como el conjunto de asociaciones voluntarias que no son parte del Estado y sin embargo ejercen alguna forma de poder social, los partidos políticos, los movimientos ciudadanos, los medios de comunicación, la empresa privada, los gremios, los sindicatos, las iglesias y las ONG (Programa de las Naciones Unidas para el Desarrollo [PNUD], 2003, p. 447).

El papel de la empresa privada como parte de la sociedad civil en el conflicto, el posconflicto y la construcción de paz ha sido objeto de diversos estudios y controversias, de las cuales se pueden destacar tres visiones: 1) su accionar puede generar mayor escalamiento e intensidad en el conflicto y por tanto es vista como una de sus causas (Swearingen, 2010; Robinson, 2013); 2) la empresa privada, en especial la local, es indispensable para la creación de empleo y oportunidades de emprendimiento, por tanto, debe ser tratada con un actor principal (Prandi y Lozano, 2010), y 3) la empresa es víctima del conflicto y la que más ha sido perjudicada como consecuencia de la guerra (Asociación Nacional de Industriales de Colombia, Consejo Internacional de Industria Sueca y Fundación Ideas para la Paz, 2014) (figura 1).

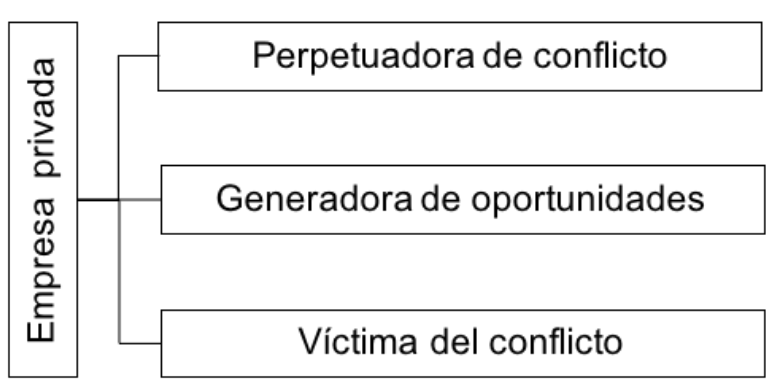

Figura 1. Enfoques de la empresa privada en el conflicto.

La primera visión considera que la empresa privada es factor clave en la generación y perpetuación del conflicto debido a que concentra el poder y riqueza en manos unos pocos y genera inequidades sociales que son principalmente el origen del conflicto (Swearingen, 2010), con el agravante de que el sistema de democracia a medias asegura a unas élites sociales la perpetuación en el poder (Robinson, 2013). Alguna información que podría corroborar esta posición muestra que Colombia es uno de los países con mayor crecimiento y desarrollo en la región en los últimos años (Banco Mundial, 2014); pero, en contraste, es uno de los que tiene mayor desigualdad social (PNUD, 2014) y mayor tasa de desempleo (Comisión Económica para America Latina y E1 Caribe [Cepal], 2014a). 
Otra situación es que las guerrillas o los grupos armados al margen de la ley se financian a través de las llamadas vacunas o contribuciones de las empresas a cambio de permitir su funcionamiento y no afectar su infraestructura (Swearingen, 2010). La contribución financiera se convierte en combustible del conflicto, aunque se sabe que en general se hace bajo amenazas de sufrir atentados contra su infraestructura, recurso humano y cadena productiva.

Desde esta visión la empresa puede contribuir a construir la paz fortaleciendo acciones de responsabilidad social y devolviendo a la sociedad parte de sus utilidades como retribución por permitir la explotación de una actividad económica. Por su parte, el Estado debe generar políticas de inclusión social en las que la riqueza y el desarrollo logren llegar a todos los ciudadanos y elimine las causas del conflicto.

La segunda visión de la empresa identifica un potencial papel en la construcción de paz, en especial desde lo local, como generadora de oportunidades de empleo y emprendimiento para las personas con menores posibilidades, desmovilizados y víctimas de la guerra (Prandi y Lozano, 2010).

Colombia es quizá el único país en el mundo que habiendo guerra interna tiene una economía emergente y una estructura empresarial sólida, hasta el punto de poder ser la tercera economía de América Latina (Cepal, 2014a), con presencia de grandes multinacionales locales y extranjeras, varias de ellas con más empleados que la totalidad de las guerrillas (Fundación Ideas Para la Paz, 2015). Estas condiciones hacen creer que de producirse un cese a la violencia el PIB podría multiplicarse por dos (Schippa, 2010). Por ejemplo, si en 2014 la economía del país hubiese crecido 8.4 \%, sería una de las economías de mayor crecimiento en el mundo.

El empleo en Colombia lo produce el sector informal en $49 \%$, la empresa privada formal $42.8 \%$ y el sector público $8.2 \%$ (Guevara, 2003), es decir, las oportunidades de empleo las genera el sector privado en $91.8 \%$. Desde esta visión la empresa privada es el principal socio que tiene la construcción de paz, lo cual genera empleo y oportunidades en especial en lo local, donde las personas no tienen espacios para su desempeño en las entidades estatales.
Otras formas para crear oportunidades y acciones de construcción de paz es aprovechando la experiencia de las grandes empresas con la asesoría a proyectos productivos, el apadrinamiento de pymes, la generación de pasantías o prácticas laborales y la compra de productos o servicios (PNUD, 2006) a aquellas microempresas o famiempresas que surjan a partir del proceso de paz y que puedan convertirse, además, en generadores de empleo para reinsertados.

La tercera visión consiste en ver la empresa como víctima, que es obligada a pagar extorsiones, chantajes para poder trabajar y comercializar bienes y servicios en determinados territorios con el agravante de que la las organizaciones prefieren no denunciar, ya que el costo puede ser mucho mayor en términos económicos, de seguridad y retaliaciones, que puedan tomar los grupos armados (Asociación Nacional de Industriales de Colombia, Consejo Internacional de Industria Sueca y Fundación Ideas para la Paz, 2014). Igualmente, la empresa es víctima en cuanto debe soportar pérdidas económicas a causa de baja productividad causada por daños en la infraestructura vial, eléctrica y de comunicación. "Moverse en un entorno impregnado de violencia representa en sí costos sustanciales y reales que la sociedad se ve obligada a asumir" (Álvarez y Rettberg, 2008, p. 32).

Si pudiera documentarse lo que pierde el sector privado a causa de los conflictos armados y lo que ganaría si hubiera paz, las organizaciones que miran por sus propios intereses preferirían invertir tiempo y dinero en la construcción de paz, en lugar de soportar la incertidumbre de los mercados, la devastación de recursos que afecta el comercio y el intercambio de bienes y servicios (Rettberg, 2010). Por esas consideraciones, la empresa privada se convierte en el principal socio estratégico de la sociedad y el Gobierno para construir la paz.

Las tres perspectivas exigen que la empresa privada sea considerada como un actor influyente en la etapa del posconflicto y la construcción de paz, de manera que coadyuve a la creación de escenarios factibles para el desarrollo económico del país y a la estabilidad social, entendiendo que no es la única responsable y su esfuerzo aislado no puede dar los resultados que se requieren, por lo que es necesaria la cohesión con el Estado, los actores armados, los organismos nacionales e internacionales
Empresa privada: principal socio en el posconficto y la construcción de paz

paz

$+2$


que propicien un entendimiento y le permitan desempeñar eficientemente su rol.

Julio Alfonso

González

Mendoza I

El sector privado sabe que las condiciones de la economía podrían llegar a ser muy favorables en un país estable, seguro y en paz, donde las oportunidades de negocios se podrían multiplicar. La empresa entiende el rol que debe desempeñar, pero desconoce o desconfía de las herramientas para contribuir al proceso y teme que esa responsabilidad la termine asumiendo en su totalidad con los consecuentes costos económicos y políticos (Velazco, 2006). Por ello, en esta sección, se reflexiona sobre los instrumentos que el empresario tiene para participar en un proceso complejo, en el que la sociedad civil debe estar comprometida.La literatura da cuenta de tres instrumentos mediante los cuales el sector privado puede participar activamente en la construcción social: 1) como financiadora de construcción de paz y posconflicto dada su capacidad económica (Kolk y Lenfant, 2013); 2) mediante alianzas estratégicas con el Estado y otras empresas nacionales o multinacionales aprovechando su credibilidad, liderazgo, experiencia y capacidad para adelantar acciones que trascienden en la sociedad (Abramov, 2010), y 3) a través de la responsabilidad social empresarial como contraprestación ética y moral a la sociedad por permitir su accionar (Jiménez, 2006).

Como financiadora de construcción de paz, la forma más expedita y clara es a través del pago de impuestos, ya que el esfuerzo económico, en últimas, representaría una inversión necesaria para ampliar los negocios y tener mayor probabilidad de expansión. Los empresarios estarían dispuestos a pagar, incluso, algún gravamen adicional o a explorar una reforma tributaria con el fin de lograr el propósito de la paz (Dinero, 2012).

Panorama I pp. 84-92। Volumen 10 I Número 18। Enero-junio I 2016 I Si se tiene en cuenta que el país cuenta con una de las más altas tasas de tributación en el mundo, $75.4 \%$ en 2014 (Banco Mundial, 2014), es necesario considerar que, pese a la voluntad de los empresarios y su deseo de tener un ambiente estable y seguro, su competitividad puede verse afectada por efecto de las cargas impositivas que ya están pagando y puedan pagar adicionalmente por la coyuntura del proceso de paz.
Si bien los empresarios quieren hacer esta apuesta y algunos están dispuestos a aportar inclusive un poco más, no se puede asfixiar la economía y cargarla de toda responsabilidad financiera y poner en riesgo su competitividad. Por tanto, podrían adoptarse otras medidas complementarias, como la lucha contra la evasión, la corrupción y el contrabando, además de apelar a la responsabilidad de las empresas para evitar estas prácticas nocivas.

El segundo mecanismo disponible es a través de las alianzas estratégicas con el Estado y las ONG, que cuenta con el antecedente de que Colombia ya tiene una tradición de alianzas público-privadas para alcanzar fines económicos y sociales. Diversas son las iniciativas de este tipo que se han establecido para desarrollar proyectos de emprendimiento, fortalecimiento a cadenas productivas, desarrollo de mercados, creación de empleo $\mathrm{y}$ formación profesional a personas en condiciones de vulnerabilidad.

Como ejemplo de estos convenios, se pueden mencionar las alianzas que el Gobierno sueco, el Gobierno colombiano y la empresa privada han establecido a través de las fundaciones Asociación Nacional de Industriales de Colombia, Ruta Motor y Fondo de Innovaciones para la $\mathrm{Paz}$, con el fin de promover proyectos productivos, creación de empleo y cadenas de valor (Embajada de Suecia en Bogotá, 2015).

Sin embargo, y pese a que muchas de estas iniciativas son exitosas, no todas las empresas tienen el conocimiento o hacen parte de alianzas. Un estudio realizado por la Cámara de Comercio de Bogotá (2015) encontró que $91 \%$ de las empresas no cuenta con proyectos que contribuya a la construcción de paz, 80 \% no conoce los programas y $80 \%$ sí estaría dispuesto a participar en alguno de ellos.

Las alianzas estratégicas y la cooperación con fines de paz son lideradas por parte de la Agencia Presidencial de Cooperación Internacional de Colombia (2014), que tiene como objetivo gestionar, orientar y coordinar la cooperación internacional pública, privada, técnica y financiera que reciba y otorgue el país. Este organismo, además de caracterizar y coordinar los cooperantes, ha creado mecanismos, manuales y procedimientos para acceder a la cooperación para la construcción de paz. 
Puede afirmarse entonces que el Gobierno y los gremios económicos tienen una importante tarea para hacer que todo el sector productivo conozca las herramientas y pueda vincularse activamente, ya que, como se dijo, existe la voluntad expresa para aportar, pero no se tiene el conocimiento ni la orientación necesaria.

El tercer mecanismo con el que cuenta la empresa privada para ayudar a la construcción de la paz es la responsabilidad social empresarial, definida por Vargas (2014) "como el conjunto de responsabilidades que las empresas asumen de manera voluntaria debido a las demandas y expectativas de sus grupos de interés o stakeholders" (p. 27). Son voluntarias, porque no se originan en las normas legales sino en demandas de clientes, propietarios, accionistas, trabajadores, comunidades, medios de comunicación, organizaciones sociales e incluso del Gobierno, aunque no sea obligatorio.

Dentro de la responsabilidad social empresarial existen otras herramientas específicas que ayudan al empresario a construir acciones sociales y reconstrucción de paz: 1) el Pacto Global de la Organización de las Naciones Unidas, 2) la guía ISO 26000, 3) las directrices de la Organización para la Cooperación y el Desarrollo Económicos (OCDE) para empresas multinacionales, 4) la iniciativa global de reportes, 5) los principios voluntarios de seguridad y derechos humanos y 6) los principios rectores de la Organización de las Naciones Unidas sobre empresas y derechos humanos (Vargas, 2014).

El Pacto Global es una iniciativa de las Naciones Unidas que promueve la responsabilidad social de las empresas y se compromete a respetar sus principios, representados en derechos humanos, medio ambiente, estándares laborales y anticorrupción (Jiménez, 2014). Colombia ha avanzado en esa dirección, ya que la Asociación Nacional de Industriales de Colombia, que es el gremio económico más representativo de la plataforma productiva colombiana, en 2005 se adhirió a esa red y espera que la iniciativa logre que la responsabilidad social sea más eficiente y desarrolle acciones concretas de construcción de paz.

Con respecto a la guía ISO 26000, es el instrumento que ofrece recomendaciones más detalladas sobre el comportamiento de las organizaciones en relación con la prevención de la violencia directa y la protección de los derechos humanos. El documento recomienda a las empresas establecer una política de derechos humanos, ser particularmente cuidadosas en situaciones de riesgo para los derechos humanos, evitar complicidad asegurando que el personal de seguridad respete los derechos humanos y disponer de mecanismos de resolución de reclamaciones (International Organization for Standardization [ISO], 2010).

La tercera herramienta que facilita el cumplimiento de responsabilidad social empresarial son las Lineas directrices de la OCDE para empresas multinacionales (OCDE, 2011), que son aplicables por parte de compañías multinacionales que operen en los países miembros de esa organización y en otros que deseen adherirse, recordando que Colombia está en el proceso de ingreso en el organismo. Las Lineas directrices de la OCDE están enfocadas para que las empresas propendan al respeto de los derechos humanos, respeto de las normas del derecho internacional humanitario, lo cual puede ayudar a las empresas a evitar impactos negativos a la sociedad (Vargas, 2014).

En el mismo sentido, las herramientas de la Iniciativa del Reporte Global, los Principios Voluntarios de Seguridad y Derechos Humanos y los Principios Rectores de la ONU sobre las Empresas y los Derechos Humanos ofrecen recomendaciones e instrucciones para favorecer la protección de los derechos humanos y encaminar esfuerzos a minimizar el impacto negativo del accionar de las empresas hacia la sociedad.

Como se aprecia, hay diferentes mecanismos disponibles para trascender en la sociedad realizando acciones de responsabilidad social empresarial, que logren mitigar los impactos negativos del actuar de las organizaciones y devuelvan a las comunidades parte de las utilidades como contraprestación por la explotación de una actividad económica. Sin embargo, algunas estadísticas muestran que estas herramientas no se conocen o no se implementan por parte de los empresarios.

Por ejemplo, un estudio realizado en el municipio de los Patios (Norte de Santander) por el programa Cercapaz encontró que apenas $37.75 \%$ de las empresas conoce el tema de responsabilidad social empresarial y que después de haber realizado talleres pedagógicos y de sensibilización los empresarios están dispuestos a adoptar esos programas en sus compañías, eso sí trabajando en cohesión con otros actores, en especial con la tutoría de
Empresa privada: principal socio en el posconficto y la construcción de paz

\section{paz}

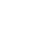


los Gobiernos nacional y regional (Ospina, Cárdenas y Beltrán, 2008) (figura 2).

Julio Alfonso

González Mendoza I
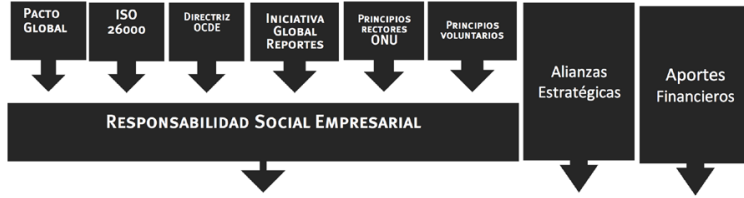

EMPLEO, ASESORIA A PROYECTOS PRODUCIVOS; APADRINAMIENTO DE PYMESS GENERACIÓN DE
PASANTASS O PRACTICAS LABORALS; COMPRA DE PRODUCTOS O SERVICIOSA LOS PEQUENOS

PASANTIAS O PRACTICAS LABORALES; COMPRA DE PRODUCTOS O SERVICIOS A LOS PEQUENOS
PRODCTORE.

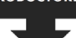

\section{CONSTRUCCION DE PAZ}

Figura 2. Herramientas de la empresa privada para la construcción de paz.

Desde luego que estos mecanismos no son nuevos y las empresas, en especial las grandes, los han ido adoptando, pero el ideal es que todo el sector productivo los conozca y los adopte con confianza y se comprometa en la construcción de un país más equitativo y libre de causas que perpetúen el conflicto, que a la vez generen nuevas oportunidades de negocio y mayor desarrollo de la sociedad.

\section{CONCLUSIONES}

Lograr el fin del conflicto no solo es poner fin a la confrontación armada, sino tomar una serie de medidas sobre las estructuras sociales que eliminen las causas de la guerra, produzcan reglas aceptadas por los ciudadanos y garanticen la solución de sus diferencias sin recurrir a la violencia. Este proceso es denominado construcción de paz y, además, se caracteriza porque la ayuda no solo es la entrega de bienes y servicios, sino una actuación ética, que no discrimine a los destinatarios y reivindique la igualdad entre los seres humanos.

El esfuerzo para la construcción de paz es responsa-

La empresa privada es considerada un actor protagónico, no solo por sus fines altruistas y de responsabilidad social, sino porque prefiere invertir tiempo y dinero en la construcción de paz, en lugar de soportar la incertidumbre de los mercados, la devastación de recursos que afecta el comercio, la extorsión, los secuestros, la ausencia de inversión en infraestructura y los ataques por parte de los grupos armados.

El sector privado reconoce su rol y responsabilidad, pero desconoce las formas para canalizar su esfuerzo, teme que esa responsabilidad la termine asumiendo en su totalidad, cree que el Gobierno envía mensajes contradictorios o divergentes, le preocupa que con estos procesos se premie a las personas que han estado al margen de la ley, percibe que el Gobierno solo lo tiene en cuenta cuando enfrenta problemas financieros y siente que no se le explica lo suficiente sobre cómo se invierten los recursos aportados.

Para generar confianza y canalizar los esfuerzos del sector privado, el Estado ha elaborado una serie de mecanismos que permiten desarrollar acciones específicas, como generación de empleo directo, identificación y asesoría a proyectos productivos, apadrinamiento de pymes, generación de pasantías o prácticas laborales, compra de productos o servicios provistos por beneficiarios del programa, implementación de proyectos productivos, etc.

Son tres los mecanismos principales con los cuales los empresarios contribuyen al proceso de construcción de paz: 1) como financiadoras de construcción de paz y posconflicto dada su capacidad económica; 2) alianzas estratégicas con el Estado y otras empresas nacionales o multinacionales dada su credibilidad, liderazgo, experiencia y capacidad de adelantar acciones que trasciendan la sociedad, y 3) a través de responsabilidad social empresarial con lo cual contribuye a la construcción de paz como contraprestación ética y moral a la sociedad por permitir su accionar.

\section{REFERENCIAS}

1. Abramov, I. (2010). Building peace in fragile states-Building trust is essential for effective public-private partnerships. Journal of Business Ethics, 89(4), 481-494.

2. Agencia Presidencial de Cooperación Internacional de Colombia (2014). Caracterización de actores de la cooperación internacional en Colombia. Recuperado de https://www.apccolombia.gov.co/recursos_user/ Documentos/Caracterizacion-de-UsuariosAPC-Colombia-22052014.pdf 
3. Álvarez, S. y Rettberg, A. (2008). Cuantificando los efectos económicos del conflicto: una exploración de los costos y los estudios sobre los costos del conflicto armado colombiano. Colombia Internacional, 67, 14-37.

4. Asociación Nacional de Industriales de Colombia, Consejo Internacional de Industria Sueca y Fundación Ideas para la Paz (2014). Como construir la paz desde el sector empresarial en Colombia. Bogotá.

5. Banco Mundial (1961-2014). Crecimiento del PIB ( $\%$ anual). Recuperado de http://datos. bancomundial.org/indicador/NY.GDP.MKTP. $\mathrm{KD} . \mathrm{ZG}$

6. Barreto Henriques, M. (2009). El Laboratorio de Paz del Cauca y Nariño: ¿una salida indígena para la paz en Colombia? En J. A Restrepo y D. Aponte (eds.), Guerra y violencias en Colombia: herramientas e interpretaciones (pp. 545-585). Bogotá: Pontificia Universidad Javeriana.

7. Cámara de Comercio de Bogotá (2015).

Encuesta de Percepción de Seguridad Empresarial 2015. Módulo de paz. Recuperado de http://bibliotecadigital.ccb.org.co/bitstream/handle/11520/13342/Encuesta \%20 de \%20Percepci \%C3 \%B3n \%20Empresarial. pdf? sequence $=3 \&$ is Allowed $=y$

8. Comisión Económica para América Latina y el Caribe (Cepal) (2014a). Estudio económico de América Latina y el Caribe 2014: desafíos para la sostenibilidad del crecimiento en un nuevo contexto externo. Santiago de Chile: Naciones Unidas. Recuperado de http://repositorio. cepal.org/bitstream/handle/11362/36970/ S1420392_es.pdf?sequence $=1$

9. Comisión Económica para America Latina y El Caribe (Cepal) (2014b). Anuario Estadístico de América Latina y el Caribe. Santiago de Chile: Naciones Unidas. Recuperado de http://interwp. cepal.org/anuario_estadistico/anuario_2014/ pdf/anuarioestadisticoalc-2014.pdf

10. Dinero (2012). Más impuestos para conseguir la paz. Recuperado de http:// www.dinero.com/pais/articulo/ mas-impuestos-para-conseguir-paz/158239

11. Drucker, P. F. (2002). Escritos fundamentales. Buenos Aires: Sudamericana.

12. Embajada de Suecia en Bogotá (2015). Alianzas con el sector privado. Recuperado de http:// www.swedenabroad.com/es-ES/Embassies/ Bogota-DC/Cooperacion-para-el-desarrollo/ Sector-privado-sys/

13. Fundacion Ideas para la Paz (2015). Los empresarios y la paz. Recuperado de http://www. ideaspaz.org/tools/download/73430
14. Garzón Galiano, J., Parra González, A. del P. y Pineda Neisa, A. S. (2003). El posconflicto en Colombia: coordenadas para la paz (Tesis de grado, Pontificia Universidad Javeriana, Bogotá, Colombia).

15. Guevara Fléchter, D. A. (2003). Globalización y mercado de trabajo en Colombia: algunas consideraciones en el marco de la flexibilización laboral. Reflexión Política, 5(10), 102-114.

16. International Organization for Standardization (2010). ISO 26000 Guidance on Social Responsibility. Ginebra: International Standard Organization.

17. Jiménez Peña, G. (2006). Más allá de la responsabilidad social empresarial: hacia un punto de vista de la ciudadanía corporativa deliberativa. Vox Populi, 5(1), 11-25.

18. Jiménez Peña, G. (2014). Multinacionales y responsabilidad social empresarial en la construcción de paz en Colombia. Cuadernos de Administración, 27(48), 67-96.

19. Kolk, A. y Lenfant, F. (2013). Multinationals, CSR and partnerships in Central African conflict countries. Corporate Social Responsibility and Environmental Management, 20(1), 43-54.

20. Organización de las Naciones Unidas (ONU) (1992). Un programa de paz, diplomacia preventiva, establecimeinto de la paz y mantenimiento de la paz. Recuperado de http://www.un.org/es/ comun/docs/?symbol=A/47/277

21. Ospina, A., Cárdenas, C. y Beltrán, M. (2008). ¿Cómo la responsabilidad social empresarial aporta a los objetivos del milenio? Guía práctica para los empresarios. Bogotá: GTZ.

22. Organización de Cooperación y Desarrollo Económico (OCDE) (2013). Lineas directrices de la OCDE para empresas multinacionales. OECD Publishing.

23. Prandi, M. y Lozano, J. M. (eds.) (2010). La responsabilidad social empresarial en contextos de conflicto y postconflicto: de la gestión del riesgo a la creación de valor. Barcelona: Escola de Cultura de Pau (UAB), Instituto de Innovación Social (ESADE).

24. Presidencia de la República (2012). II laboratorio de paz: principales resultados y aprendizajes. Recuperado de http://www.eeas. europa.eu/delegations/colombia/documents/ projects/20110909_es.pdf

25. Programa de las Naciones Unidas para el Desarrollo (PNUD) (2003). Informe nacional de desarrollo bumano para Colombia 2003. Madrid: Mundi-Prensa.
I Panorama

I pp. 84-92

I Volumen 10 
26. Programa de las Naciones Unidas para el Desarrollo (PNUD) (2006). Los empresarios y la reinserción: un reto mayor. Hechos El Callejón, 22(18), 2-5.

Julio Alfonso

González

Mendoza I

Panorama I

pp. 84-92।

Volumen 10 I

Número 18 ।

Enero-junio I

2016 |
27. Programa de las Naciones Unidas para el Desarrollo (PNUD) (2014). Informe sobre desarrollo humano 2014. Sostener el progreso humano: reducir vulnerabilidades y construir resiliencia. Nueva York. Recuperado de http://hdr.undp.org/ sites/default/files/hdr14-summary-es.pdf

28. Rettberg, A. (2003). Diseñar el futuro: una revisión de los dilemas de la construcción de paz para el posconflicto. Revista de Estudios Sociales, $15,15-28$.

29. Rettberg, A. (2010a). De los confictos armados a la construcción de paz. La participación del sector privado en la construcción de paz: inventario e identificación de algunos ejemplos ilustrativos. Barcelona: Universidad Ramon Llull.

30. Rettberg, A. (2010b). La participación del sector privado en la construcción de paz: inventario e identificación de algunos ejemplos ilustrativos. Barcelona: Escola de Cultura de Pau (UAB), Instituto de Innovación Social (ESADE).

31. Robinson, J. A. (2013). Colombia: Another 100 years of solitude? Current History, 112(751), 43.

32. Schippa, C. (2010). El valor de la paz para la economía. Barcelona: Universidad Ramon Llull.

33. Swearingen, M. (2010). Group inequality and conflict: Some insights for peacebuilding. Washington: United States Institute of Peace.

34. Universidad del Rosario (2014). Experimentos sobre reconciliación política en Colombia. Recuperado de http://www.urosario.edu.co/ jurisprudencia/jurisprudencia-reconciliacion/

35. Universidad Jorge Tadeo Lozano (2012). Empresa y la construcción de paz. Cuadernos $\mathrm{Paz}$ a La Carta, 3, 1-18.

36. Vargas, G. A. (2014). La responsabilidad social empresarial en la construcción de paz: una introducción. Documentos en Desarrollo Cider, 4. Bogotá: Universidad de los Andes.

37. Velazco, M. (2006). Participación del sector empresarial en la reinserción: percepciones y oportunidades. Bogotá: Fundación Ideas para La Paz. 